\title{
Management of a patient diagnosed with COVID-19 who underwent double valve replacement: A Case Report.
}

\author{
Aysen Engin ${ }^{1}$, Onur Saydam ${ }^{1}$, and Cengiz Sert ${ }^{1}$ \\ ${ }^{1}$ Izmir Tepecik Training and Research Hospital
}

May 14, 2020

\begin{abstract}
Abstract: The number of infected cases with COVID-19 increase gradually, and some of the COVID-19 patients present with concomitant cardiovascular problems that might require surgical intervention. However, we do not have sufficient evidence of optimum timing for cardiac surgery in patients with COVID-19 whose surgery cannot be postponed or who cannot be treated percutaneously. Here we discuss the management of a patient that diagnosed with COVID-19 and required urgent cardiac surgery for symptomatic moderate aortic and severe mitral valve regurgitation.
\end{abstract}

Title: Management of a patient diagnosed with COVID-19 who underwent double valve replacement: A Case Report.

Running title: Management of COVID-19 heart valve regurgitation

Authors: Aysen Yaprak Engin ${ }^{1}$, Onur Saydam ${ }^{1}$, Cengiz Sert ${ }^{1}$

Institutions and Affiliations: 1 Tepecik Training and Research Hospital, Guney mahallesi 1140/1 sokak no: 1 yenisehir - konak - Izmir / Turkey, Department of Cardiovascular Surgery

Classifications: Case Report

Corresponding Author: Onur Saydam, Musa Anter Sokak No:65 Mavisu Evleri Sitesi No: 34b D:17 Narlidere / Izmir, 35320 Telephone: +90(533)357 09 23, onursaydam@hotmail.com

Abstract: The number of infected cases with COVID-19 increase gradually, and some of the COVID-19 patients present with concomitant cardiovascular problems that might require surgical intervention. However, we do not have sufficient evidence of optimum timing for cardiac surgery in patients with COVID-19 whose surgery cannot be postponed or who cannot be treated percutaneously. Here we discuss the management of a patient that diagnosed with COVID-19 and required urgent cardiac surgery for symptomatic moderate aortic and severe mitral valve regurgitation.

Keywords: COVID-19; aortic valve regurgitation; mitral valve regurgitation; aortic valve replacement; mitral valve replacement

Introduction

Novel coronavirus (COVID-19) has been declared as a worldwide pandemic by the World Health Organization and at the time of writing this case report, there are over 3,250,000 reported cases worldwide and over 120,000 cases in Turkey. ${ }^{1}$ COVID-19 is a highly contagious respiratory virus which is mainly transmitted by droplets and contact. Symptomatic patients may present with fever, dry cough, and shortness of breath. ${ }^{2,}{ }^{3}$ The number of infected cases with COVID-19 increase gradually, and some of the COVID-19 patients present with concomitant cardiovascular problems that might require surgical intervention. However most of the 
cardiovascular surgery units faced a sudden change in their daily practice and limit their surgery to only selected emergency cases with selected patients. In order to avoid the additional burden of cardiac surgery and reduce the length of stay in hospital, patients who need to go to surgery under normal conditions are being postponed or diverted to the cardiology department for percutaneous interventions, if possible. However, we do not have sufficient evidence of optimum timing for cardiac surgery patients with COVID-19 in whom surgery cannot be postponed or who cannot be referred to percutaneous interventions. Here we discuss the management of a patient who was diagnosed with COVID-19 and required urgent cardiac surgery for symptomatic moderate aortic valve regurgitation (AR) and severe mitral valve regurgitation (MR).

\section{Case Report}

A fifty-five-year-old male patient was admitted to the emergency room with New York Heart Association (NYHA) Class IV congestive heart failure symptoms. Physical examination revealed bibasal crackles in the chest, pansystolic murmur at the apex of the heart and severe bilateral pretibial edema. The patient had a history of hypertension and he had been on treatment with metoprolol $50 \mathrm{mg}$ twice daily, perindopril $10 \mathrm{mg}$ once daily and furosemide $40 \mathrm{mg}$ on alternate days for 1 year due to mild mitral and aortic valve regurgitation. Dyspnea had started 2 months ago and its severity gradually increased. Electrocardiography showed sinus tachycardia with 120 beats per minutes. He had no signs of fever or dry cough. Afterwards, the patient was referred to cardiology clinic and transthoracic echocardiography (TTE) demonstrated a left ventricle ejection fraction of $35 \%$ and his left atrial diameter was $45 \mathrm{~mm}$, left ventricle end diastolic diameter was $57 \mathrm{~mm}$ and systolic pulmonary artery (sPAB) pressure was $60 \mathrm{mmHg}$. He had severe MR and moderate AR. His laboratory values were unremarkable except for increased d-dimer levels $(840 \mathrm{mcg} / \mathrm{l}$, reference range: $0-450 \mathrm{mcg} / \mathrm{l}$ ), impaired lipid profile and a slightly increased urea level. As the patient had normal cardiac enzyme levels and a history of a recent coronary angiography, repeat of coronary angiography was not planned by cardiology. Thereafter the patient was referred to cardiovascular surgery department and surgery was planned due to severe symptoms. Although the patient had symptoms of heart failure and was diagnosed with MR and AR the routine screening was performed in emergency department for COVID-19. The patients who is transferred from emergency department to the other clinics with one or more COVID-19 symptoms are screened for COVID-19, as Turkey is among the countries with the highest number of COVID 19 cases. Respiratory samples are obtained from both nasopharyngeal swab and sputum sample. Reverse transcription polymerase chain reaction test (RT-PCR) are performed for diagnosis. Because test result can be obtained up to 24 hours, the patient was followed by strict droplet and contact precautions in a negative air-flow Intensive Care Unit (ICU) room considered as a potential COVID-19 positive case. Test was resulted positive for COVID-19. The patient's history was thoroughly questioned and was learned that there was no history of upper respiratory tract infection or fever recently. Serum procalcitonin level was in normal range. Computed tomography (CT) scan of the chest was normal (Figure 1). Several consultations to related departments including infectious diseases, chest diseases and cardiology was performed to determine the optimum timing of surgery. By the decision taken, the surgery was postponed until the test result becomes negative. According to the treatment protocol of the Republic of Turkey Ministry of Health for COVID-19 patients, hydroxychloroquine $200 \mathrm{mg}$ twice daily was initiated. Fever, dry cough or worsening of dyspnea was not observed during follow-up. Therefore, hydroxychloroquine was ceased on the $5^{\text {th }}$ day of its onset. RT-PCR test was repeated once every two days. PCR-RT test was found to be negative on the day $10^{\text {th }}$ and $11^{\text {th }}$. Posterior anterior chest $\mathrm{x}$ ray and TTE was repeated on the day $14^{\text {th }}$ and showed parallel results with the previous tests. D-dimer level was regressed to $490 \mathrm{mcg} / \mathrm{l}$. When his clinical status was concerned and after a multi-disciplinary consultation and shared decision making of the risks and benefits with the patient, it was determined that he should undergo cardiac surgical intervention.

Surgery was scheduled. All of the staff who involved in the operation used appropriate protective equipments (PPE) including; N-95 mask, face shield, gown, and gloves. During left atrium exposure, significant degenerative MR was detected. Depending on the condition of the valve, it was decided to replace the valve by the surgeons involved in the surgery. Therefore, mitral valve was replaced with a 31-mm mechanical valve. Aortic valve was also replaced with a $21-\mathrm{mm}$ mechanical valve (St. Jude Medical, Inc., Little Canada, Minnesota, USA). No perioperative or postoperative excessive bleeding was detected. The extubation of the 
patient was performed 6 hours after the patient's arrival in intensive care. Postoperative posterior anterior chest X-ray revealed a pleural effusion, along with atelectasis in the left lower lobe (Figure 2a). Control TTE revealed a left ventricle ejection fraction of $45 \%$ and mitral valve prosthetics were in normal function. Mean trans-mitral gradient was $4 \mathrm{mmHg}$, mean trans-aortic gradient was $15 \mathrm{mmHg}$ and sPAB was $25 \mathrm{mmHg}$. Anticoagulation was initiated with enoxaparin (Clexaneß, Sanofi Aventis, Guildford, United Kingdom) in the early postoperative period and replaced with Warfarin in the following days. After two days in the intensive care unit patient was transferred to inpatient service of cardiovascular surgery. The patient was discharged on postoperative day 6 under warfarin treatment with NYHA class 1 symptoms.

\section{Discussion}

To the best of our knowledge, we present the first case of a patient diagnosed with COVID-19 and underwent successful mitral and aortic valve replacement after recovery from COVID-19. COVID-19 outbreak was first detected in Wuhan, in Hubei province of China on December 12, 2019 and recently declared as a "pandemic" by the World Health Organization. ${ }^{4}$ Turkey became one of the most affected countries according to the number of confirmed COVID-19 patients at the time of writing this case report. Due to sudden increase in number of critically ill COVID-19 patients, increasing demand for ICU beds, ventilators and physicians became a common problem in Europe. Therefore many cardiovascular ICU's were converted to COVID-19 critical care units and many cardiac surgeons find themselves working as a COVID-19 doctor. However, there is an estimated 420 million patients diagnosed with cardiovascular disease and nearly 18 million people die due to cardiovascular diseases a year. ${ }^{5}$ Massive demand is still present today, and patients with cardiovascular diseases who are diagnosed with COVID 19 also appear with an increasing number. Although there is no treatment protocol for COVID-19 diagnosed cardiovascular patients who needs surgery, today mostly two alternative methods are being used; postponing the operation or diverting the patients to the percutaneous interventions. Both alternatives are controversial, because postponing a surgery may risk the patient's life and long-term outcomes of forced percutaneous interventions may end up with undesired results. There are also patients who are not suitable for both options. In our case report, we present the management of case of COVID-19 diagnosed patient with symptomatic severe mitral and moderate aortic regurgitation. Although, minimal invasive treatment choices can be performed such as transcatheter mitral valve repair using an edge-to-edge clip for MR and transcatheter aortic valve implantation for pure AR, surgery is still the gold standard. ${ }^{6,7}$ As a cardiovascular surgeon, we have experience in preoperative, perioperative and postoperative management in patients with viral infections such as hepatitis and HIV infection, however, currently we do not have enough experience with COVID-19 infected patients. Not all COVID-19 patients have the similar symptoms, the clinical spectrum of COVID-19 ranges from asymptomatic to critically ill cases. ${ }^{8}$ The study conducted by Xu et al, ${ }^{9}$ among asymptomatic COVID-19 patients, only (6.7\%) showed leukopenia. Most of the patients' whole blood counts were in normal range and $84.6 \%$ of patients had normal serum procalcitonin on admission. Majority of the patients had normal D-dimer levels (92.7\%). In parallel, the patient in our report also had normal blood count and procalcitonin levels, however the patient's D-dimer levels was increased. Although, the RT-PCR is considered as the reference standard test, disadvantages of RT-PCR including difficulties during sample collection and initial false negative results lead physicians to use of chest $\mathrm{CT}$ as an initial diagnostic tool. ${ }^{10} \mathrm{In}$ the early stages, thorax $\mathrm{CT}$ findings including bilateral pneumonia and ground glass opacity could be present even before the onset of symptoms. ${ }^{11}$ However, only $53.4 \%$ of the asymptomatic patients have abnormalities in the chest CT examinations on admission. ${ }^{9}$ Similarly, the patient in our report also had a normal chest CT examination.

The patient was diagnosed with COVID-19, but since he was asymptomatic, it was not known how old his disease was. COVID-19 may have a more severe prognosis in patients with cardiovascular diseases and the course of the disease can be changed in the following days. ${ }^{12}$ Therefore, the patient's surgery had to be postponed and close follow-up in intensive care unit was performed in order to avoid additional cardiac and pulmonary burden to the patient. During the follow-up, hydroxychloroquine $200 \mathrm{mg}$ twice daily was initiated and continued for 5 days. Republic of Turkey Ministry of Health has recommended hydroxychloroquine for patients diagnosed with COVID-19. ${ }^{13}$ Hydroxychloroquine is mainly an anti-malarial drug which also has an indication outside of COVID-19 such as rheumatoid arthritis, and systemic lupus erythematosus. A 
study conducted by Gautret ${ }^{14}$ et al showed that hydroxychloroquine treatment is associated with viral load reduction and disappearance in COVID-19 patients. In contrast, some authors are more cautious about this treatment approach due its' negative effects including prolong the QT interval and induce arrhythmias. ${ }^{15}$ No electrocardiographic changes were detected during follow-up. This may explain as the adverse effects of this drug mostly occurs in long-term use and in cases where the daily dose is more than $400 \mathrm{mg} .{ }^{16} \mathrm{PCR}$ RT test was found negative on the $10^{\text {th }}$ day and repeated tests were also negative on the $11^{\text {th }}$. Because the patient was asymptomatic for COVID-19 symptoms, the test dates were established according to the incubation period of the disease based upon preliminary reports. ${ }^{17}$ However, there is still lack of data about exact incubation period and it may be longer. ${ }^{18}$ Therefore, all of the staff who involved in the operation and during the follow in ICU used appropriate protective equipment (PPE).

Whenever possible, mitral valve repair is preferred over mitral valve replacement for mitral regurgitation. However, anatomical status of the valve plays a major role, and, in our case, mitral valve replacement was performed due to highly degenerative anatomical status of the mitral valve. Although some of the highly degenerative mitral valves may also be repaired, the repair may cause prolonged operation time or reoperation in the early period. In addition, in case of early failure, mechanical aortic valve would have further complicate mitral valve replacement in the early reoperation. In both cases the risk for myocardial and pulmonary injury due to cardiopulmonary bypass may increase. ${ }^{19,}{ }^{20}$ Indeed, it has been found that, COVID-19 infects host cells through angiotensin-converting enzyme 2 (ACE2) receptors and this receptors are densely found in the heart and lungs which can cause to pneumonia and/or acute myocardial injury. ${ }^{21}$ Although the patient had no findings indicating COVID-19 in imaging methods and all cardiac and pulmonary findings were thought to be related to severe mitral regurgitation, there is still lack of data whether chronic cardiac or pulmonary issues take place even in patients with negative test results. In the control TTE performed in the postoperative period, an improvement in the cardiac functions of the patient was observed which precluded us from the fact that preoperative heart failure could be due to COVID-19. There was no delay in extubation time. During the patient's postoperative intensive care process, no additional pulmonary support was needed. The patient was transferred to inpatient clinic on postoperative day 2 and discharged on postoperative day 6 .

Inconclusion

Open heart surgery may be performed safely in patients who previously had asymptomatic COVID-19 under certain precautionary rules when tests become negative. While creating this result, this patient group should be closely followed-up after the procedures for complications and outcomes with large randomize control trials.

Funding statement: Authors certify that no funding has been received for this study

Conflict of interest statement: All authors certify that they have no conflicts of interest in the research.

Authors contribution statement: All authors meet the following criterias including; substantial contributions to the conception or design of the work; or the acquisition, analysis, or interpretation of data for the work, drafting the work or revising it critically for important intellectual content, final approval of the version to be published, agreement to be accountable for his/her contributions of the work in ensuring that questions related to the accuracy or integrity of the work are appropriately investigated and resolved.

\section{References}

1. World Health Organization Coronavirus disease 2019 (COVID-19): situation reporthttps://www.who.int/docs/defaultsource/coronaviruse/situation-reports/20200330-sitrep-70-covid-19. ; 2020 .

2. Moriarty LF, Plucinski MM, Marston BJ, Kurbatova EV, Knust B, Murray EL, et al. Public Health Responses to COVID-19 Outbreaks on Cruise Ships - Worldwide, February-March 2020. MMWR Morb Mortal Wkly Rep. 2020;69(12):347-52.

3. Zhu N, Zhang D, Wang W, Li X, Yang B, Song J, et al. A Novel Coronavirus from Patients with 
Pneumonia in China, 2019. N Engl J Med. 2020;382(8):727-33.

4. Xiao Y, Torok ME. Taking the right measures to control COVID-19. Lancet Infect Dis. 2020.

5. Watkins DA, Johnson CO, Colquhoun SM, Karthikeyan G, Beaton A, Bukhman G, et al. Global, Regional, and National Burden of Rheumatic Heart Disease, 1990-2015. N Engl J Med. 2017;377(8):713-22.

6. Bonow RO, O'Gara PT, Adams DH, Badhwar V, Bavaria JE, Elmariah S, et al. 2020 Focused Update of the 2017 ACC Expert Consensus Decision Pathway on the Management of Mitral Regurgitation. J Am Coll Cardiol. 2020.

7. Arias EA, Bhan A, Lim ZY, Mullen M. TAVI for Pure Native Aortic Regurgitation: Are We There Yet? Interv Cardiol. 2019;14(1):26-30.

8. Liang WH, Guan WJ, Li CC, Li YM, Liang HR, Zhao Y, et al. Clinical characteristics and outcomes of hospitalised patients with COVID-19 treated in Hubei (epicenter) and outside Hubei (non-epicenter): A Nationwide Analysis of China. Eur Respir J. 2020.

9. Xu T, Huang R, Zhu L, Wang J, Cheng J, Zhang B, et al. Epidemiological and clinical features of asymptomatic patients with SARS-CoV-2 infection. J Med Virol. 2020.

10. Gunduz Y, Ozturk MH, Tomak Y. The usual course of Thorax CT findings of Covid-19 infection and When should control Thorax CT scan? Turk J Med Sci. 2020.

11. Guneyli S, Atceken Z, Dogan H, Altinmakas E, Atasoy KC. Radiological approach to COVID-19 pneumonia with an emphasis on chest CT. Diagn Interv Radiol. 2020.

12. Li B, Yang J, Zhao F, Zhi L, Wang X, Liu L, et al. Prevalence and impact of cardiovascular metabolic diseases on COVID-19 in China. Clin Res Cardiol. 2020;109(5):531-8.

13. The Republic of Turkey Ministry of Health General Directorate of Public Health Guidance to Covid-19 (SARS CoV 2 infection) (scientific board study)https://hsgm.saglik.gov.tr/depo/birimler/goc_sagligi/covid19/rehber/English-COVID-19-Guide-14April2020.pdf; 2020.

14. Gautret P, Lagier JC, Parola P, Hoang VT, Meddeb L, Mailhe M, et al. Hydroxychloroquine and azithromycin as a treatment of COVID-19: results of an open-label non-randomized clinical trial. Int J Antimicrob Agents. 2020:105949.

15. Fihn SD, Perencevich E, Bradley SM. Caution Needed on the Use of Chloroquine and Hydroxychloroquine for Coronavirus Disease 2019. JAMA Netw Open. 2020;3(4):e209035.

16. Stokkermans TJ, Trichonas G. Chloroquine And Hydroxychloroquine Toxicity. StatPearls. Treasure Island (FL)2020.

17. Li Q, Guan X, Wu P, Wang X, Zhou L, Tong Y, et al. Early Transmission Dynamics in Wuhan, China, of Novel Coronavirus-Infected Pneumonia. N Engl J Med. 2020;382(13):1199-207.

18. Lauer SA, Grantz KH, Bi Q, Jones FK, Zheng Q, Meredith HR, et al. The Incubation Period of Coronavirus Disease 2019 (COVID-19) From Publicly Reported Confirmed Cases: Estimation and Application. Ann Intern Med. 2020.

19. Zupancich E, Paparella D, Turani F, Munch C, Rossi A, Massaccesi S, et al. Mechanical ventilation affects inflammatory mediators in patients undergoing cardiopulmonary bypass for cardiac surgery: a randomized clinical trial. J Thorac Cardiovasc Surg. 2005;130(2):378-83.

20. Wan S, DeSmet JM, Barvais L, Goldstein M, Vincent JL, LeClerc JL. Myocardium is a major source of proinflammatory cytokines in patients undergoing cardiopulmonary bypass. J Thorac Cardiovasc Surg. 1996;112(3):806-11. 
21. Zheng YY, Ma YT, Zhang JY, Xie X. COVID-19 and the cardiovascular system. Nat Rev Cardiol. 2020;17(5):259-60.

Figure Legends

Figure 1: Initial chest tomography: No definitive signs of COVID was detected.

Figure 2a: Preoperative posterior anterior chest X-ray

Figure 2b: Postoperative posterior anterior chest X-ray; The sternal metal suture wires was seen, pleural effusion, along with atelectasis in the left lower lobe
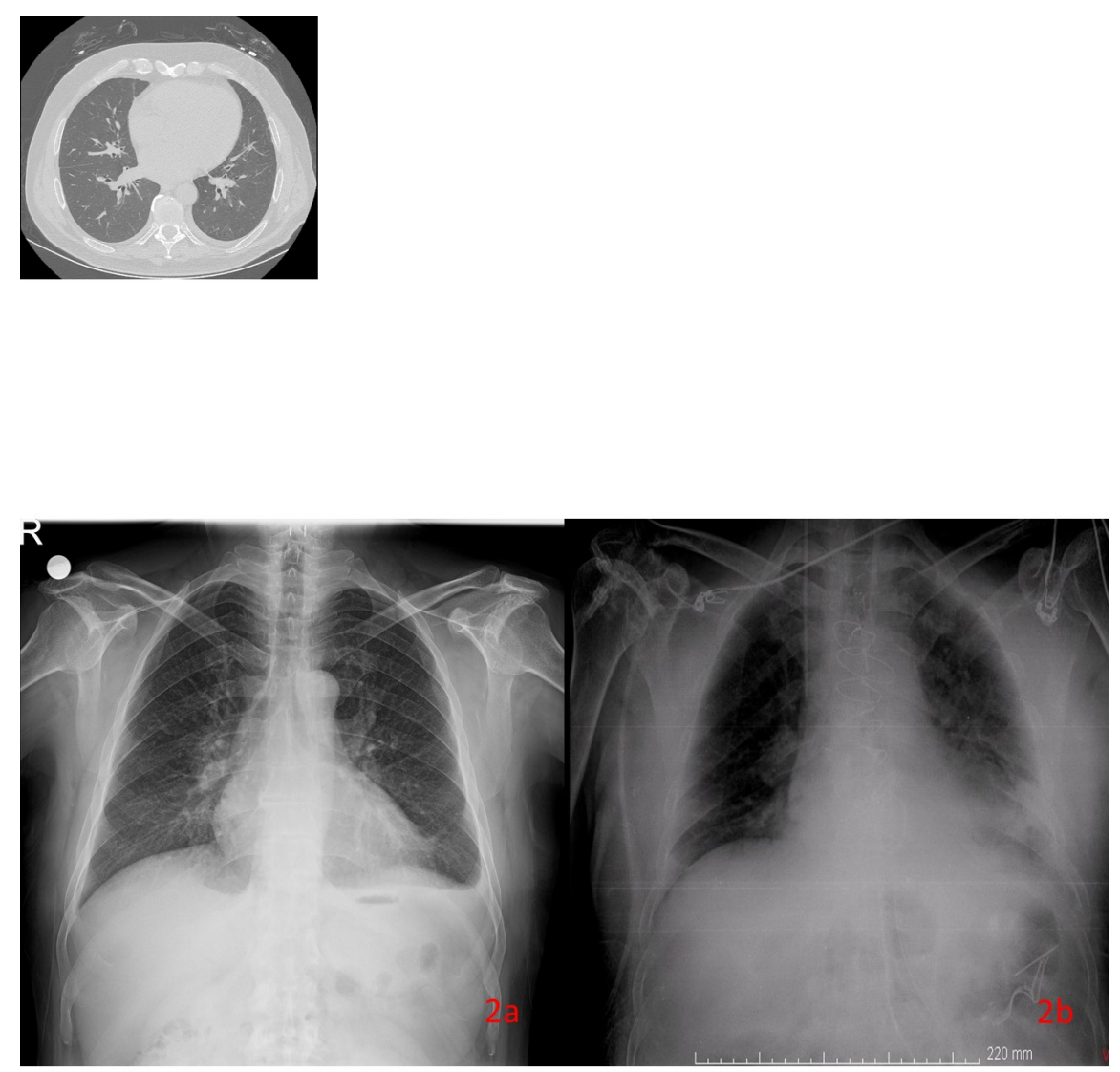